УДК 621.382

\title{
В.Л. Самотовка
}

Национальный технический университет Украины «Киевский политехнический институт», ул. Политехническая, 16, каб.419, Киев, 03056, Украина.

\section{Оценка качества фротоэлектрических преобразователей на структурах с p-n переходом}

В работе представлены результаты исследований кремниевых фротоэлектрических преобразователей с различными значениями коэфрфициентов полезного действия и фракторов заполнения. Приводятся теоретически полученные выражения параметров качества, основанные на применении модифицированных фрункций Бесселя, аргументы в которых пропорциональны температуре, m-фрактору $p$ - $n$ перехода и амплитудным значениям, применяемых разночастотных гармонических напряжений. Приводится интегральный показатель качества, являющийся наибольшим значением напряжения разностной частоты при нагрузках равных эффрективному сопротивлению фотоэлектрической структуры с p-n переходом. Библ. 7, рис. 1, табл. 2.

Ключевые слова: эфрфрективное сопротивление, разностная частота, КПД, модифицированные фрункции Бесселя, фоотоэлетрические преобразователи.

\section{Введение}

Фотоэлектрические преобразователи (ФЭП) на основе полупроводниковых структур с p-n переходом (ПС), в сравнении с параметрическими диодами, характеризуются низкими значениями активного последовательного и шунтирующего параллельного, относительно p-n перехода, сопротивлений. Это обусловлено относительно большой площадью и минимальновозможным расстоянием p-n перехода к освещаемой поверхности пластины. В работе [1] указано о несущественном влиянии шунтирующего статического активного сопротивления на эффективность ФЭП. Однако при различных нагрузочных сопротивлениях ширина p-n перехода может меняться, вовлекая в область пространственного заряда различное количество структурных дефектов. Это может влиять на величину дифференциального шунтирующего сопротивления.

В работах $[2,3,4,5,6]$ представлены методы измерения диффференциальных и статиче- ских, шунтирующих p-n переход сопротивлений, но не рассмотрена возможность определения шунтирующих сопротивлений в диапазоне напряжений прямого смещения p-n перехода. В данной работе представлены методы и результаты определения шунтирующих p-n переход сопротивлений в режиме прямого смещения p-n перехода и отсутствия светового потока.

\section{Модифицированные функции Бесселя в дифференциальной спектроскопии электри- ческого тока в элементе с p-n переходом}

Электрическая цепь (ЭЦ), которая применялась для определения параметров ФЭП, представлена на (рис. 1). Где 1-безинерционная электрическая цепь, состоящая из последовательного соединения ПС-2 и линейного нагрузочного резистора $R_{i}$; 3-p-n переход; $V$ напряжение, прикладываемое к ЭЦ; $R_{s h^{-}}$шунтирующее $\mathrm{p}-\mathrm{n}$ переход сопротивление; $R_{s^{-}}$ последовательное активное сопротивление.

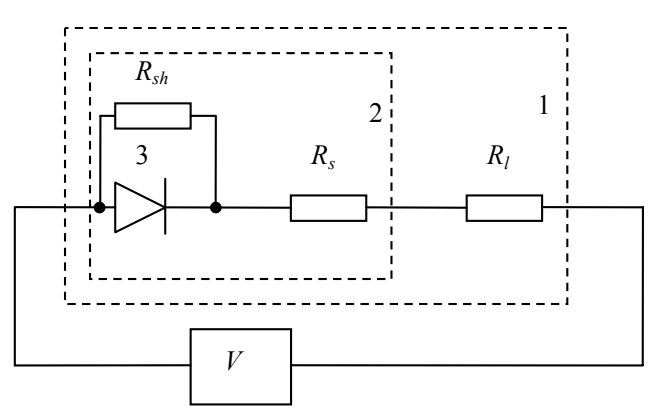

Рис. 1. Структурная схема ЭЦ

Напряжение приложенное к ЭЦ состоит из постоянного напряжения прямого смещения $V_{0}$ и двух малосигнальных гармонических напряжений на разых частотах $\omega_{1}$ и $\omega_{2}$ :

$V=V_{0}+V_{1 m} \cos \omega_{1} t+V_{2 m} \cos \omega_{2} t=V_{0}+V_{1}+V_{2}$ (1)

Статическую ВАХ ПС ФЭП при постоянном напряжений $V_{0}$ в интервале напряжений, определяемом суммой гармонических напряжений

(с) Самотовка В.Л., 2014 
$V_{1}+V_{2}, \quad$ аппроксимируем кусочноэкспоненциальной функцией:

$$
i_{0}=i_{s}\left(e^{a\left(V_{0}-i_{0} R\right)}-1\right)+\frac{V_{0}-i_{0} R}{R_{s h}},
$$

где $R=R_{s}+R_{;} ; a=q /(k T m) ; R_{s}$-сопротивление полупроводниковой структуры последовательно соединенное с p-n переходом; $R_{s h}$-сопротивление параллельное p-n переходу ФЭП.

Используя выражения для постоянного и гармонических токов [7], протекающих в ЭЦ при условии $R_{s}=0 \quad R_{s h} \rightarrow \infty R_{F}=0$, протекающих в ЭЦ рис. 1 при действии на нее напряжения (1) получены максимальные значения токов на разностной $\omega_{1} \pm \omega_{2}$ и удвоенной $2 \omega_{1}$ частотах, которые достигаются при постоянном напряжении прямого смещения p-n перехода:

$$
\begin{gathered}
V_{0 d m}\left(\omega_{1} \pm \omega_{2}\right)=\frac{1}{a} \ln \left(\frac{R+R_{s h}}{8 a k_{11}\left(y_{1}\right) k_{12}\left(y_{2}\right) i_{s} R R_{s h}}\right) \\
I_{\omega 1 m}=\frac{J_{02}\left(y_{2}\right)}{J_{02}\left(y_{2}\right)+4 k_{12}\left(y_{2}\right)} \times \\
\times \frac{V_{1 c}}{R}+\frac{4 k_{12}\left(y_{2}\right)}{J_{02}\left(y_{2}\right)+4 k_{12}\left(y_{2}\right)} \cdot \frac{V_{1 c}}{R+R_{s h}} \\
I_{\omega 1 \pm \omega 2}=\frac{2 \sqrt{2} k_{11}\left(y_{1}\right) k_{12}\left(y_{2}\right) a^{2} i_{s} e^{a\left(V_{0}-i_{0} R\right)} V_{1 c} V_{2 c}}{\left(1+R \sigma_{s h}+4 k_{11}\left(y_{1}\right) k_{12}\left(y_{2}\right) R a i_{s} e^{a\left(V_{0}-i_{0} R\right)}\right)^{3}}
\end{gathered}
$$

Если $R_{s h} \gg R$, то выражение для сопротивления $R_{s}$ используя (3-5) можно выразить:

$$
R_{\mathrm{s}}=\varepsilon \cdot \frac{V_{1 c}}{I_{\omega 1 m}}-R_{l}=\varepsilon \frac{V_{1 c}}{V_{\omega 1 m}} R_{l}-R_{l}
$$

где:

$$
\varepsilon=\frac{J_{02}\left(y_{2}\right)}{J_{02}\left(y_{2}\right)+4 k_{12}\left(y_{2}\right)} .
$$

Постоянное напряжение прямого смещения p-n перехода, при котором достигается максимальное значение тока разностной частоты $V_{0 d m}$, ток насыщения $i_{s}$ и $m$-фрактор BAX определяют из соотношений:

$$
V_{0 d m}\left(\omega_{1} \pm \omega_{2}\right)=V_{0 m}-\varepsilon \cdot \frac{V_{1 c}}{I_{\omega 1 m}} \cdot i_{0 m}
$$

$i_{s}=$

$$
\begin{gathered}
=\left(54 \sqrt{2} \varepsilon^{2} k_{11}\left(y_{1}\right) k_{12}\left(y_{2}\right) \frac{V_{1 c}}{V_{2 c}} \frac{I_{(\omega 1-\omega 2) m}}{l_{\omega 1 m}^{2}} e^{a V_{0 d m}(\omega 1-\omega 2)}\right)^{-1} \\
m=\frac{2 \sqrt{2}}{27} \frac{q V_{2 c}}{k T \varepsilon} \frac{I_{\omega 1 m}}{I_{(\omega 1-\omega 2) m}} .
\end{gathered}
$$

Из (4) получили выражения для шунтирующего p-n переход сопротивления $R_{s h}$ :

$$
R_{s h}=\frac{\left(R_{l}+R_{s}\right)^{2}-(\varepsilon+\eta)\left(R_{l}+R_{s}\right) R_{e f 1}}{R_{e f 1} \varepsilon-\left(R_{l}+R_{s}\right)}
$$

где:

$$
\eta=\frac{4 k_{11}\left(y_{2}\right)}{J_{01}\left(y_{2}\right)+4 k_{12}\left(y_{2}\right)}, \quad R_{e f 1}=\frac{V_{1 c}}{I_{\omega 1 m}}=\frac{V_{1 c}}{V_{\omega 1 m}} R_{l} .
$$

Если аргументы $y_{1}$ и $y_{2}$ фуннкции Бесселя меньше 0,35 , то с погрешностью менее $1 \%$ можно принять, что $\varepsilon=1 / 3$, а $\eta=2 / 3$. Тогда шунтирующее $\mathrm{p}-\mathrm{n}$ переход сопротивление:

$$
R_{s h}=\frac{3\left(\left(R_{l}+R_{s}\right) R_{e f 1}-\left(R_{l}+R_{s}\right)^{2}\right)}{3\left(R_{l}+R_{s}\right)-R_{e f 1}}
$$

Кроме того, с учетом указанных значений $\varepsilon=1 / 3$ и $\eta=2 / 3$, если не выполняется условие $R_{s h}$ >> $3\left(R_{l}+R_{s}\right)$, то $m$-фрактор:

$$
\begin{aligned}
& m=\frac{2 \sqrt{2}}{9} \frac{q V_{2 c}}{k T} \frac{R_{s h}^{2}}{\left(3\left(R_{l}+R_{s}\right)+R_{s h}\right) \cdot\left(R_{l}+R_{s}+R_{s h}\right)} \times \\
& \times \frac{I_{\omega 1 m}}{I_{(\omega 1-\omega 2) m}} .
\end{aligned}
$$

\section{Экспериментальная часть}

Исследования проведены на образцах кремниевых фотоэлектрических преобразователей на структурах c p-n переходом. Образцы характеризуется значением фактора заполнения (FF) и коэффрициентом полезного действия. Фотоэлектрические параметры образцов ФЭП представлены в табл. 1.

Таблица 1. Параметры ФЭП

\begin{tabular}{|c|l|l|l|l|}
\hline \multirow{2}{*}{$\begin{array}{l}\text { № об- } \\
\text { разца }\end{array}$} & \multicolumn{1}{|l|}{ Параметры ФЭ } & V0c, B & $\begin{array}{l}\text { КПД, } \\
\%\end{array}$ & FF \\
\cline { 2 - 5 } & Isc & \\
\hline 1 & 3,265 & 0,604 & 6,0 & 47,0 \\
\hline 2 & 5,497 & 0,629 & 15,6 & 69,8 \\
\hline 3 & 5,573 & 0,628 & 17,3 & 76,5 \\
\hline 4 & 5,6 & 0,63 & 18,2 & 82,0 \\
\hline
\end{tabular}

Каждый ФЭП включали в электрическую цепь согласно рисунку 1 вместо ПС. В отсутствии светового потока на ЭЦ подавали напряжение (1). Устанавливали различные значения сопротивления нагрузочного регулируемого резистора $R_{/}$. Для каждого фиксированного значения сопротивления $R_{l}$ изменяли величину постоянного напряжения $V_{0}$ до уровня $V_{0 m}$, при котором ток разностной частоты $I_{\omega 1-\omega 2}$ достигает максимального значения. На резисторе $R_{l}$ измеряли падение напряжения $V_{\omega 1 m}$, созданное током $I_{\omega 1 m}$, напряжения $V_{(\omega 1-\omega 2) m}$, созданное током $I_{(\omega 1-\omega 2) m}$ и постоянное напряжение $V_{0 m}$, приложенное к ЭЦ. Среднеквадратичные значения гармонических напряжений $V_{1 c}$ и $V_{2 c}$ и соответствующие им амплитудные значения $V_{1 m}, V_{2 m}$ выбраны из условий $y_{1} \leq 0,35 ; y_{2} \leq 0,35$.

Расчет шунтирующего сопротивления $R_{s h}$ выполнен, используя (13), $R_{s}$ (6) и m-фрактор (10). $R_{s}$ и $m$-фрактор определяли при сопротив- 
лении нагрузки 0,1 Ом. Результаты исследований приведены в таблице 2. В результате анализа полученных данных выявлен наиболее информативный интегральный параметр качества ПС ФЭП - $\lambda$ :

$V_{(\omega 1-\omega 2) m}=\frac{2 \sqrt{2}}{27} \cdot \frac{q}{k T m} \cdot \frac{R_{s h}^{2} V_{1 c} V_{2 c} R_{l}}{R\left(R+R_{s h}\right)^{2}} \sim \lambda=$

$=\left(m\left(R_{l}+R_{s}\right)\left(1+\frac{R_{l}+R_{s}}{R_{s h}}\right)^{2}\right)^{-1}$.

Он объединяет максимальное значение падения напряжения разностной частоты $V_{(\omega 1-\omega 2) m}$ на фиксированном сопротивлении 0,1 Ом резисто-

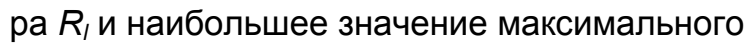

напряжения $V_{(\omega 1-\omega 2) m}^{\max }$ и тока $I_{(\omega 1-\omega 2) m}^{\max }$ разностной частоты в диапазоне нагрузочных сопротивлений 0,1-0,9 Ом. Указанный интегральный параметр качества включают такие известные показатели качества ПC, как шунтирующее p-n переход сопротивление $R_{s h}$, последовательно соединенное с p-n переходом сопротивление $R_{s}$ и $m$ фактор. Кроме того, выявлен такой параметр, как максимальное значение сопротивления нагрузочного резистора $R_{/ c}$. Увеличение сопротивления нагрузочного резистора выше $R_{/ c}$ приводит к росту постоянного напряжения $V_{0 m}$ и снижению $R_{s h}$. Таким образом, более высокое значение $R_{/ c}$ характеризует более качественные ПС ФЭП.

Таблица 2. Результаты эксперимента

\begin{tabular}{|c|c|c|c|c|c|c|c|c|c|c|c|c|}
\hline $\begin{array}{l}\text { № } \\
\text { обр. }\end{array}$ & $\begin{array}{l}\mathrm{RI}= \\
0,1 \Omega, \\
\mathrm{V}(\omega 1- \\
\omega 2) \mathrm{m}, \\
\mu \mathrm{V}\end{array}$ & $\begin{array}{l}\mathrm{V} \\
\max \\
(\omega 1- \\
\omega 2) \mathrm{m}, \\
\mu \mathrm{V}\end{array}$ & $\begin{array}{l}\mathrm{m}, \\
\mathrm{Rl}= \\
0,1 \\
\Omega\end{array}$ & $\begin{array}{l}\text { 2RImax, } \\
\Omega\end{array}$ & $\begin{array}{l}\operatorname{Imax}(\omega 1- \\
\omega 2) \mathrm{m}, \\
\mu \mathrm{A}\end{array}$ & $\begin{array}{l}\text { Rshmax, } \\
\Omega\end{array}$ & $\begin{array}{l}\text { Rlc, } \\
\Omega\end{array}$ & $\begin{array}{l}\text { Rshc, } \\
\Omega\end{array}$ & $\begin{array}{l}\text { V0m, } \\
\text { V }\end{array}$ & $\begin{array}{l}\text { Rs, } \\
\Omega\end{array}$ & $\mathrm{m}$ & $\begin{array}{l}\lambda, \\
\Omega-1\end{array}$ \\
\hline 1 & 170 & 190 & 1,51 & 0,9 & 422 & 7,0 & 4,0 & 12 & 0,45 & 0,052 & 1,7 & 1,02 \\
\hline 2 & 216 & 256 & 1,29 & 0,4 & 1270 & 3,5 & 0,4 & 4,5 & 0,53 & 0,01 & 1,36 & 3,12 \\
\hline 3 & 260 & 282 & 1,2 & 0,4 & 1410 & 6,0 & 6,5 & 11 & 0,46 & 0,012 & 1,25 & 3,39 \\
\hline 4 & 300 & 307 & 1,3 & 0,25 & 2456 & 18 & 7,0 & 20 & 0,45 & 0,01 & 1,25 & 5,63 \\
\hline
\end{tabular}

\section{Заключение}

Таким образом, в данной работе выведены теоретические выражения для расчета показателей качества ПС ФЭП таких, как $R_{s}, R_{s h}, m-$ фрактора и $i_{s}$, с учетом влияния значений аргументов функций Бесселя. Предложен выбор значений аргументов, при которых функциями Бесселя можно пренебречь. Выявлены показатели качества ПС ФЭП такие, как максимальное значение падения напряжения разностной частоты $V_{(\omega 1-\omega 2) m}$ на фриксированном сопротивлении, наибольшее значение максимального тока разностной частоты и критическое сопротивление нагрузочного резистора $R_{/ c}$.

\section{Список использованных источников}

1. С. Зи. Физика полупроводниковых приборов.-М.:Мир, 1984.-Т. 2.-456 с.

2. M.-K. Lee, J.-C. Wang, S.-F. Horng, H.-F. Meng, "Extractoin of solar cell series resistance without presumed current-voltage function form," Solar Energy Materials \& Solar Cells, vol. 94, pp. 578-582, March 2010.

3. D. Cotfas, P. Cotfas, S. Kaplanis, D. Ursutiu, "Results on series and shunt resistance in a CSi PV cell. Comparison using existing methods and a new one," Journal of optoelectronics and advanced materials, vol. 10, \#11, pp. 31243130, November 2008.

4. J. Thongpron, K. Kirtikara, C. Jivacate, "A method for the determination of dynamic resistance of photovoltaic modules under illumination," Solar Energy Materials \& Solar Cells, vol. 90, pp. 3078-3084, November 2006.

5. D.L. King, B.R. Hansen, J.A. Kratochvil and M.A. Quintana, "Dark current-voltage measurements on photovoltaic modules as a diagnostic or manufacturing tool," 26th IEEE Photovoltaic Specialists Conference, pp. 11251128, September 29-October 3, 1997, Anaheim, California.

6. M. Wolf and H. Rauschenbach, "Series resistance effects on solar cell measurements," Advanced energy conversion, vol. 3, pp. 455-479, Pergamon Press, Great Britain, 1963.

7. Самотовка Л.И., Самотовка В.Л. Математическое моделирование токов полупроводниковой структуры с p-n переходом.// Электроника и связь. Тематический выпуск "Проблемы электроники".-2008.-ч.1.-с.38-42.

Поступила в редакцию 20 сентября 2014 г.

(C) Самотовка В.Л., 2014 
УДК 621.382

В.Л. Самотовка

Національний технічний університет України «Київський політехнічний інститут», вул. Політехнічна, 16, каб.419, Київ, 03056, Україна.

\section{Оцінка якості фотоелектричних перетворювачів на структурах з p-n переходом}

В роботі наводяться результати досліджень кремнієвих фоотоелектричних перетворювачів з різними значеннями коефріцієнтів корисної дії та фракторів заповнення. Також наводяться теоретичні вирази для параметрів якості, які отримуються з використанням модифікованих фрункцій Бесселя, аргументи в яких пропориійні температурі, m-фрактору p-n перехода та амплітудним значенням, різночастотних гармонічних напруг. Пропонується інтегральний показник якості, який є найбільшим значенням напруги різницевої частоти коли навантаження равні ефрективному опору фоотоелектричної структури з р-п переходом. Бібл. 7, рис. 1, табл. 2

Ключові слова: ефективний опір, різницева частота, ККД, модифріковані фуннкції Бесселя, фотоелектричні перетворювачі.

\section{UDC 621.382}

\section{Samotovka}

The National Technical University of Ukraine "Kyiv Polytechnic Institute", st. Polytechnique, 16, off. 419, Kyiv, 03056, Ukraine.

\section{Quality assessment of photovoltaic cells with p-n junction}

In this work research results for photovoltaic cells with different efficiencies and fill factors are given. Perfection factors' expressions are theoretically derived using modified Bessel functions. Bessel functions have arguments that proportional to temperature, $p$ - $n$ junction's $m$ factor and amplitudes of used harmonic voltages with different frequencies. It is determined that the integral perfection index is the most differential frequency voltage's value measured when load is less than effective resistance of photovoltaic structure with p-n junction. References 7 , figures 1 , tables 2.

Keywords: effective resistance, differential frequency, efficiency factor, modified Bessel functions, photovoltaic cells.

\section{References}

1. Cotfas D., Cotfas P., Kaplanis S., Ursutiu D. (2008), "Results on series and shunt resistance in a c-Si PV cell. Comparison using existing methods and a new one". Journal of optoelectronics and advanced materials, vol. 3, \# 11, pp. 3124-3130.

2. King D.L., Hansen B.R., Kratochvil J.A., Quintana M.A. (1997), "Dark current-voltage measurements on photovoltaic modules as a diagnostic or manufacturing tool“. " $26^{\text {th }}$ IEEE Photovoltaic Specialists Conference.», pp. 1125-1128.

3. Lee M. K., Wang J. C., Horng S. F., Meng H. F. (2010), "Extractoin of solar cell series resistance without presumed current-voltage function form". Solar Energy Materials \& Solar Cells, vol. 94, pp. 578582.

4. Thongpron J., Kirtikara K., Jivacate C. (2006), "A method for the determination of dynamic resistance of photovoltaic modules under illumination". Solar Energy Materials \& Solar Cells, vol. 90, pp. 30783084.

5. Wolf M. and Rauschenbach H. (1963), "Series resistance effects on solar cell measurements". Advanced energy conversion, vol. 3, pp. 455-479.

6. Sze S. (1984), "Physics of semiconductor devices", Moscow: «Mir», vol. 2, p.456. (Rus)

7. Samotovka L.I., Samotovka V.L. (2008), "Mathematic modelling of currents in semiconductor structure with p-n junction" Electronics and Communications. «Problem in electronics», vol. 1, pp.38-42. (Rus) 